\title{
Implicaciones de la reforma a la Ley del Servicio Exterior Mexicano y su Reglamento
}

\author{
Ingrid Berlanga Vasile*
}

El Servicio Exterior Mexicano (SEM) es un cuerpo del Estado cuya misión es promover los intereses de México y de los mexicanos en el exterior, independientemente del partido que se encuentre gobernando. Se trata del servicio civil de carrera más antiguo de México y en sus miembros recae la responsabilidad de ejecutar la política exterior de México, conforme a los principios establecidos en la Constitución Política de los Estados Unidos Mexicanos.

En reconocimiento a la importante labor del SEM, en abril de 2018, culminó un esfuerzo originado varios años atrás que dio como resultado la adopción de la reforma a la Ley del Servicio Exterior Mexicano (LSEM) y a su Reglamento (RLSEM). El 19 de abril de 2018 se oficializó la publicación de esta Ley $^{1}$ y el 2 de noviembre de ese mismo año, de su Reglamento. ${ }^{2}$ Con dicha reforma se sentaron las bases para llevar a cabo mejoras significativas y adaptaciones de los beneficios y condiciones laborales de los miembros del SEM.

El Servicio Exterior Mexicano está compuesto por servidores públicos con capacidades y preparación académica, amplia experiencia en temas específicos, y constituyen un elemento vital e indispensable para la formulación e implementación de la política exterior mexicana. Se destacó que su importancia para el desarrollo nacional no debería soslayarse. Una política

* Encargada de la sección consular de la Secretaría de Relaciones Exteriores del gobierno de México.

1 Disponible en: http://www.diputados.gob.mx/LeyesBiblio/pdf/96_190418.pdf.

2 Disponible en: http://www.diputados.gob.mx/LeyesBiblio/pdf/regley/Reg_LSEM_021118.pdf. 
exterior con recursos humanos débiles difícilmente puede sostener los objetivos de política exterior de un Estado como México. La LSEM y el RLSEM contemplan artículos cuya aplicabilidad depende de la disponibilidad de recursos presupuestarios, lo cual escapa de jurisdicción exclusiva de la Secretaría de Relaciones Exteriores (SRE), razón por la cual fue esencial el cabildeo con la SHCP y el Congreso.

La Comisión de Personal llevó a cabo un proceso de intercambio de opiniones y propuestas de manera virtual con los miembros del SEM a través de una plataforma digital, incorporando posteriormente estos insumos en las discusiones que dicha Comisión sostenía entre sus miembros y con sus contrapartes en otras instancias involucradas.

Los artículos 1o. y 2o., inciso XI bis, de la LSEM destacan el papel de la SRE como ejecutora de la política exterior y de coordinadora de acciones con otras instancias gubernamentales, así como no gubernamentales. Ello obedece a la nueva realidad en la que múltiples actores de la sociedad llevan a cabo acciones al exterior de país que redundan en distintos tipos de oportunidades para los mexicanos.

La modificación al artículo 1-ter contempla una participación en condiciones de igualdad de género, lo cual no estaba contemplado anteriormente. Ello representa un gran avance ya que más de la mitad de los miembros del SEM son hombres, así como el 70\% de los embajadores y cónsules generales.

El diseño de un plan de carrera para los miembros del SEM fue una estrategia fundamental para hacer mejor uso de sus fortalezas y mejorar su desempeño. También contribuye a equiparlos con las herramientas requeridas para asumir cargos de mayor responsabilidad sin menoscabo de su desarrollo personal. Asimismo, coadyuva a brindar mayor certeza a la carrera diplomática en términos de traslados, duración de los cargos y uso de recursos. Se creó una Subcomisión de Análisis y Prospectiva (artículo 26 bis, LSEM), además de las ya existentes (Subcomisión de Ingreso, de Rotación, de Evaluación, de Asuntos Disciplinarios). Sus funciones no han sido muy claras, aunque la intención es que se encargue del constante mejoramiento de las condiciones laborales del SEM, así como de su profesionalización y evaluación, lo cual se lograría a través de la elaboración de un Plan de Carrera Quinquenal (artículo 33-ter, RLSEM). No obstante, hasta este momento, a más de un año de aprobada la LSEM y RLSEM, no hay claridad sobre dicho plan, sus consecuencias ni su viabilidad. Probablemente, 
debido a la coyuntura política del cambio de administración, y dado que "la Oficialía Mayor de la SRE deberá gestionar ante la Secretaría de Hacienda y Crédito Público el número de plazas que permitan facilitar el ascenso en los tiempos establecidos por el artículo 40 bis de la LSEM, así como para su adecuada implementación y sujeto a disponibilidad presupuestaria”.

Otro aspecto importante de la reforma fueron las causas de baja de los miembros del SEM. Según el artículo 53 bis anteriormente se podía causar baja únicamente por renuncia, jubilación, declaración de estado de interdicción, incumplimiento de orden de traslado, no reincorporación después de una comisión, disponibilidad o licencia a sus funciones. Con la reforma del artículo 40 bis se incluye la posibilidad de que un miembro del SEM cause baja definitiva bajo otro supuesto, a través de un filtro que no haya ascendido al rango de primer secretario en un plazo máximo de quince años o bien, que no haya ascendido al rango de consejero en un plazo de diez años desde su ascenso a primer secretario. También podrá causar baja definitiva un miembro del SEM cuya evaluación de desempeño arroje un resultado insatisfactorio. En todo caso, la persona será compensada por años de servicio. La razón por la que la discusión en torno a la reforma a este artículo fue tan controvertida se debió a que se asienta la posibilidad de que un miembro del SEM sea removido de su empleo por cuestiones que podrían ser subjetivas. Muchos de los ascensos al rango de primer secretario no son posibles debido a la falta de plazas suficientes y/o a la subjetividad con la cual algunos miembros son evaluados, por lo que se prestó a una disputa acerca de la legalidad de esta reforma. El artículo 33 duodecies del RLSEM indica que se recomendará la baja cuando un segundo secretario obtenga en tres ocasiones consecutivas una calificación insatisfactoria. ${ }^{3} \mathrm{Si}$ bien el criterio del desempeño debería permear en la decisión de que un miembro del SEM que no ha logrado el ascenso al rango correspondiente en la temporalidad establecida debería ser exclusivamente el desempeño, hay otros factores subjetivos que podrían influir en dicha decisión. Ésta deberá incluir aspectos como los motivos por los que el ascenso no fue posible, como la disponibilidad de plazas, las funciones que ha desempeñado el miembro del SEM, incluyendo su experiencia en adscripciones de vida difícil y en labores que, incluso, hayan puesto en riesgo su integridad física. Por otro

3 El artículo 58 RLSEM señala los componentes que definen un desempeño insatisfactorio del personal. 
lado, aquéllos que muestren indicios de pérdida de sentido de la vocación diplomática, compromiso y cuyo desempeño sea deficiente, deberían dejar de pertenecer a este cuerpo diplomático de cuya excelencia y entrega depende la implementación de una política exterior coherente con el interés nacional.

Es importante mencionar que los ascensos en el SEM se logran a través de concursos altamente competitivos que requieren de preparación y son celebrados cada año o dos, según la disponibilidad de recursos presupuestarios para cubrir la creación de plazas correspondientes. El mayor número de miembros del SEM tienen el rango de segundo secretario, por lo que la competencia por menos plazas es aún mayor.

Los artículos 7o. de la LSEM y 4o. del Reglamento, presentaron puntos de conflicto también, toda vez que se refiere al personal que desempeña funciones del SEM temporalmente (en un plazo máximo de seis años). Señala que dicho personal desempeñará las funciones del SEM de conformidad con los perfiles que determine la Comisión de Personal, mismos que serán publicados en el Diario Oficial de la Federación. En el correspondiente RLSEM, el artículo 4o. señala que el presidente de la República podrá nombrar personal de manera temporal. Se excluye el personal temporal externo al SEM de ocupar posiciones de jefatura de cancillería, cónsul adscrito, encargado de la sección consular y/o de administrador, las cuales únicamente podrán ser ocupadas por miembros del Servicio. El personal temporal podrá estar sujeto a exámenes que comprueben su capacidad para dicho cargo además de evaluaciones de su desempeño. No obstante, aún no han quedado muy definidos los criterios de selección del personal temporal ni la manera en que serán seleccionados aquéllos que ocuparán próximamente cargos en el exterior.

De igual manera, ahora el personal temporal o asimilado, así como los servidores públicos de la SRE que tengan una antigüedad mínima de dieciseis años en dichos supuestos o como servidores públicos, podrán ingresar al SEM como personal de carrera de la rama Diplomático-Consular con rango de consejero. Ello despertó inconformidad entre los miembros del SEM debido a la facilidad que se brinda a servidores públicos que no han pasado por el arduo proceso de ascensos y que no cuentan necesariamente con la formación requerida para ocupar cargos diplomáticos, y que evidentemente no lograron aprobar el concurso de ingreso. No obstante, hay que reconocer que la experiencia de laborar en la SRE por esos mínimos 
dieciseis años indudablemente imprime un historial valioso de experiencia en la práctica diplomática y/o consular, por lo que se podría considerar un avance en aras de la especialización del SEM, incorporando a estos servidores públicos.

La estructura salarial del SEM fue otra cuestión que reflejaba preocupación por sus miembros, toda vez que, no ha habido un aumento de sueldos en muchos años y en la mayoría de las adscripciones ha aumentado el nivel de vida. De tal suerte, el artículo 9o. de la Ley indica que la Comisión de Personal someterá a consideración del secretario un análisis sobre el costo de vida de los diversos países con miras a estabilizar el sueldo en la moneda local.

La profesionalización especializada y coherencia entre el perfil de los miembros del SEM y su adscripción es una cuestión que ha preocupado a sus miembros. En la práctica, hasta el momento de la reforma a dicho ordenamiento jurídico, los traslados se llevaban a cabo de manera aleatoria, y gran parte eran implementados gracias a influencias y a conocimiento previo del funcionario, mas no necesariamente, debido a sus capacidades específicamente adecuadas para dicho cargo. La Subcomisión de Rotación (artículo 11 bis LSEM) decidía el destino del servidor público. Para evitar esto, desde 2002 se reformó el artículo 11, en su párrafo 4, señalando que se favorecerá una equilibrada rotación del personal entre áreas geográficas diversas y de especialización temática, tomando en cuenta especialidades profesionales, el conocimiento de idiomas, la perspectiva de género y la integración familiar del personal del SEM. No obstante, esto aún no se lleva a cabo en la práctica.

Otro aspecto que coadyuva en la especialización y en la adquisición de mayores conocimientos y herramientas, para el buen desempeño del trabajo de los miembros del SEM, es permitirles asumir cargos temporales en otras dependencias y entidades de la administración pública federal, incluidos los poderes Legislativo y Judicial, además de instituciones de educación superior o en organismos internacionales. Ello era imposible antes de la reforma, ya que si un miembro del SEM tenía una oportunidad laboral atractiva debía solicitar licencia, perdiendo así su antigüedad y corriendo el riesgo de rezagar su ascenso al siguiente rango dentro del SEM. Esto constituye, sin duda, un gran avance que permite adquirir experiencia en otros ámbitos nacionales e internacionales. En este sentido, el artículo 33 sexies permite a la SRE celebrar convenios con dichas instancias bajo criterios como que 
el puesto que ocupará el miembro del SEM responda a los objetivos de la propia SRE y la política exterior, y favorezca su desarrollo profesional; es decir, que dichas funciones tengan relación con las actividades diplomáticas de México, y que dicho puesto tenga una temporalidad de al menos un año.

La LSEM también incluye nuevas estipulaciones que benefician a los miembros del SEM. Ahora también se cubrirá a los concubinos e hijos del miembro del SEM, incluidos quienes presenten una discapacidad, el importe de sus pasajes a México cada dos años, además de que gozarán de seguro médico (artículo 49). En el caso de adopción de un infante se otorga un permiso de seis semanas a las mujeres y de cinco días hábiles a los hombres con goce de sueldo. También, en la nueva Ley se autoriza la disponibilidad por segunda ocasión a miembros del SEM que requieran cuidar a descendientes o ascendientes o con condición médica comprobada (artículo 52). Asimismo, se permite por primera vez, que los cónyuges que sean miembros del SEM puedan estar adscritos a la misma representación.

La edad de jubilación del SEM se amplió de 65 a 70 años, con la reforma al artículo 55 LSEM, con lo cual se solventa una inquietud de muchos miembros que alcanzaban esa edad y aún deseaban continuar estando laboralmente activos. Sin embargo, los beneficios a los que tendrían derecho al alcanzar los 70 años son menores a los que recibirán de retirarse a los 65 .

El reto de la reforma a la Ley del SEM y su Reglamento consiste en implementar en la práctica, un sistema transparente y meritocrático. Aún persiste la necesidad de transversalizar las funciones y las acciones en las representaciones diplomáticas y consulares, disminuyendo la jerarquización extrema del SEM y abriendo paso, con humildad, a una generación más joven de diplomáticos, que se desenvuelvan en un ambiente más horizontal y con igualdad de género. La eficiencia y eficacia de la labor ardua, entregada y patriótica de los miembros del Servicio Exterior Mexicano deberá ser compensada adecuadamente con un presupuesto digno y con la consideración por parte de la sociedad mexicana a la cual representa. La política exterior de México debe apoyarse con una política interna consciente de los beneficios de contar con una sólida presencia internacional con gente a la altura de las circunstancias sirviendo al bien común de la nación. 